\title{
Full results on risks of epoetin emerge 14 years after major dialysis study
}

This News story (BMJ 2012;344:e3535, doi:10.1136/bmj.e3535) incorrectly stated that the normal haematocrit trial "formed the basis for regulatory approval" of the drug epoetin alfa. In fact, Cite this as: BMJ 2012;344:e3677 epoetin alfa had already been approved for use in the US. 